\title{
El encuentro del tejido urbano con el mar: límite, borde o interacción.
}

\author{
Ignacio Bosch Reig ${ }^{1}$, Luis Bosch Roig ${ }^{2}$, \\ Valeria Marcenac ${ }^{3}$, Nuria Salvador Luján ${ }^{4}$ \\ Instituto Universitario de Restauración del Patrimonio. Departamento de Proyectos \\ Arquitectónicos, Universitat Politècnica de València, Valencia, España \\ E-mail: 1ibosch@pra.upv.es, ${ }^{2}$ luibosro@pra.upv.es, ${ }^{3}$ marceval@pra.upv.es, ${ }^{4}$ nusalluj@arq.upv.es
}

\begin{abstract}
Resumen. El trabajo que se presenta sostiene la tesis de que la relación entre la ciudad y el mar ha ido evolucionando desde un planteamiento de limite o final del tejido urbano que se contrapone a un paisaje natural, y que por tanto suponía un acusado desencuentro entre ambos, hacia la búsqueda de mecanismos que propicien la interacción y continuidad entre artificio y naturaleza, consiguiéndose una nueva lectura de esa relación.

El análisis de quince casos de diferentes partes del mundo, ha permitido reconocer que en las diversas formas de establecer esta relación, es el concepto de "paseo marítimo" la clave fundamental que la define. Y en ella cabe destacar las diferentes herramientas paisajísticas que se han empleado en la definición de los recorridos, tensiones, flujos, secuencia de espacios, .... asi como las que permiten resaltar las diferentes miradas: desde el mar, desde la ciudad, visión parcial o de conjunto, intensa o contemplativa, veloz o reposada, ....
\end{abstract}

Palabras clave: paisaje, ciudad, naturaleza, transversalidad, regeneración urbana, ecosistema, sostenibilidad.

\section{Introducción}

Este trabajo sostiene la tesis de que la relación entre la ciudad y el mar planteada inicialmente como límite o final del tejido urbano, que quiere expresarse con autonomía en contraposición al paisaje natural, marcando con ello un acusado desencuentro entre ambos, ha evolucionado hacia la búsqueda de mecanismos que propicien la interacción y continuidad entre artificio y naturaleza, consiguiéndose una nueva lectura de esa relación.

En este encuentro de la ciudad con el mar, caben destacar al menos tres cuestiones de calado: la configuración de la estructura urbana en su encuentro con la línea de costa, la definición de la fachada marítima, y especialmente la relación del plano suelo del espacio público con el medio natural del mar.

\section{Configuración de la ciudad en su encuentro con la costa:}

Respecto de la influencia de la línea de costa en la configuración del tejido urbano de la ciudad, podemos decir que en muchos casos, la estructura de la ciudad ha respondido a

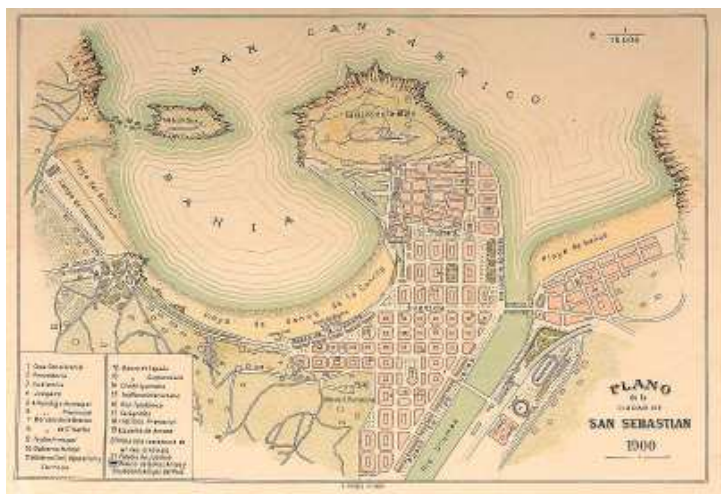

Fig. 1. Estructura Urbana de la Concha de San Sebastian. Cartografía de 1900. 

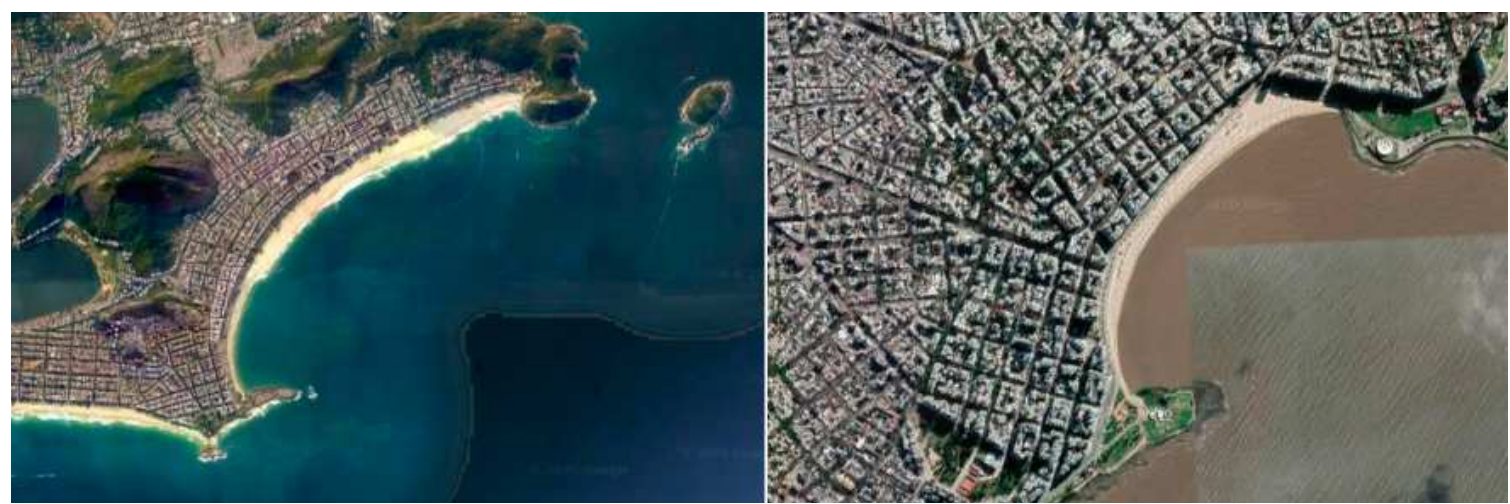

Fig. 2. Estructura urbana de Copacabana (Rio de Janeiro) y del Barrio de Pocitos (Montevideo)

condicionantes no vinculados directamente con el límite marino, como podemos apreciar en la zona de la bahía de la Concha de San Sebastián, o en la bahía de Riazor en La Coruña. (Fig. 1)

Sin embargo, en aquellas ciudades donde el tejido urbano surge como consecuencia de la necesidad de utilización de la zona costera, como es el caso del barrio de Copacabana en Rio de Janeiro (urbanización iniciada en 1898 de forma que en 1906 ya se inauguró la Av. Atlántica que bordea la bahía, ampliada en 1919), la estructura urbana evidencia una dependencia geométrica de la línea de costa, haciendo en este caso un claro ajuste de la trama ortogonal de partida, para convertirse en un sistema radial-tensional buscando la perpendicular al mar en cada manzana. (Fig. 2)

Es también el caso del barrio de Pocitos de Montevideo, cuya ordenación en cuadrícula, con manzanas de 100 varas de lado (83m), fue diseñada por el cartógrafo José María Reyes en 1833, pasando de ser una zona de recreo a finales del XIX, a consolidarse como barrio con arquitecturas de grandes casas veraniegas, entre 1915-1940, pasando en las décadas de 1950-1960, a convertirse en el barrio mas densificado y con mas calidad de vida de Montevideo. (Fig. 2)

Sin duda ello fue posible gracias a la realización de arquitecturas honestas de una factura intachable, realizadas por arquitectos reconocidos como Julio Vilamajó, Raul Sichero, Luis García Pardo, Adolfo Sommer Smit, Walter Pintos Risso, y otros muchos que supieron asimilar con sencillez y con decisión los principios de la modernidad.

La visión aérea de Pocitos, nos muestra como la densa trama, se va aproximando a la línea de la costa, ajustando, deformando y girando la cuadrícula buscando un encuentro amable con el mar.

Aquí no hay una imposición de las leyes de la ciudad posicionándose de forma autónoma con respecto al mar, sino que la Rambla, actuando de elemento intermedio entre la ordenada estructura urbana y la poderosa y cambiante naturaleza, consigue establecer una transición paisaje natural-paisaje artificial, capaz de superar el acusado desencuentro entre ambos, propiciando la interacción y la continuidad.

Estamos ante una forma de entender la estructura de la ciudad, que surge a partir de una asunción consciente de los beneficios que plantea su proximidad al mar, como son la captación del frescor de la brisa, las continuas y cambiantes vistas, la percepción de las aromas marinas, la experimentación de sensaciones diversas: dinamismo, quietud, inmensidad, ... y como no, el contacto directo con una de las fuentes de la vida, el agua, ... cuestiones todas ellas que han sido el origen de estructuras urbanas complejas, sinuosas, y claramente adaptativas, como es el caso de Venecia. (Fig. 3)

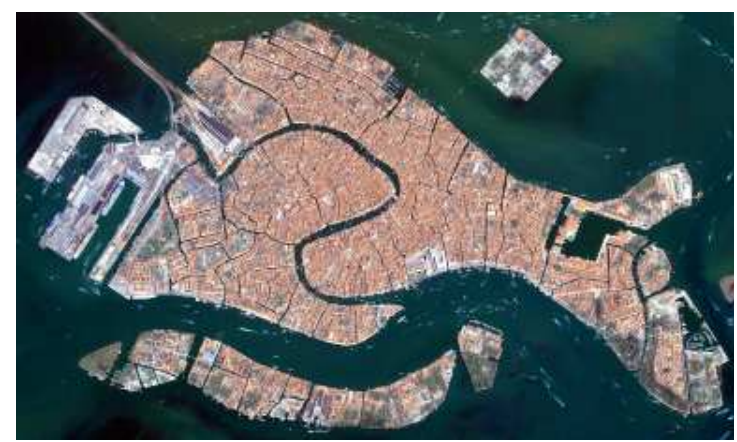

Fig. 3. Estructura urbana de Venecia 


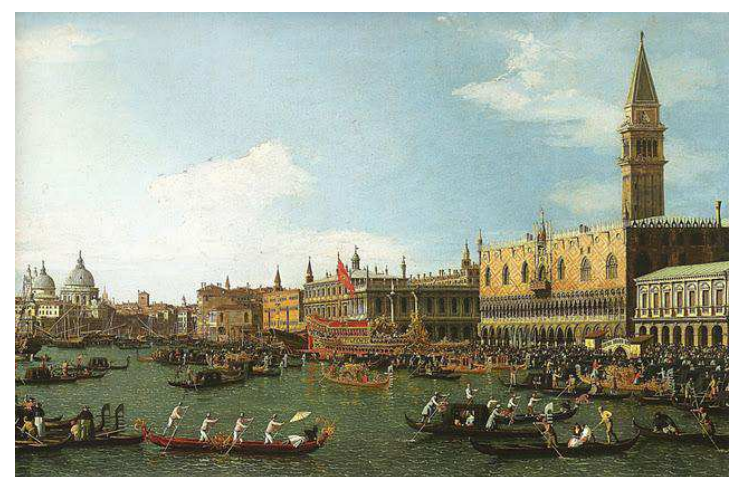

Fig. 4. Fachada Marítima de Venecia. Veduta de Canaleto, 1743

\section{Fachada Marítima:}

La segunda de las cuestiones que cabe destacar en ese encuentro del tejido urbano con el mar es la importancia de la configuración de la "Fachada marítima". Estamos ante uno de los temas mas interesantes de la configuración del paisaje urbano que no es otro que la definición del Scainline de la ciudad desde el mar.

A lo largo de la historia se ha producido de muy diversas formas, bien definiéndola de forma fragmentaria, destacando en ella las arquitecturas mas significativas, como es el caso de Venecia. (Fig. 4), bien creando una fachada continua y unitaria perfectamente ordenada, que transmite la imagen de haberse construido de una sola vez, como es el caso de las casas del muelle de la fachada marítima de Santander. (Fig. 5)

Pero esa imagen de unidad y continuidad se puede conseguir también desde la diversidad, como es el caso de Pocitos, donde los mecanismos arquitectónicos de la línea común de cornisa y del vuelo de $1,50 \mathrm{~m}$ de la totalidad la fachada de todos los edificios, junto a un sistema de orden secuencial horizontal establecido por los forjados y las terrazas, han permitido definir un frente marítimo unitario, armónico y másico, que despegándose del plano-tierra, parece querer flotar introduciéndonos en esa sugerente relación del peso y la levedad. (Fig. 5)

\section{Encuentro ciudad-mar. EI plano suelo}

La tercera de las cuestiones a resaltar del encuentro Ciudad-Mar, es la relación directa del plano suelo del espacio público urbano con el medio natural del mar..

Así en el final del siglo XIX, el tradicional uso público del litoral en España, Portugal y en los países Iberoamericanos, junto con el creciente interés por los "baños de ola", dio paso al nacimiento de los Paseos marítimos o Malecones, como paseos públicos lineales de borde, generalmente arbolados, que dieron paso a la definición de la fachada marítima, estableciéndose una cuidada imagen de la ciudad.

Esta relación se planteó inicialmente como el limite-final de la ciudad, estableciéndose como borde, como contrapunto artificio-naturaleza, a través de un planteamiento claramente invasivo y dominante de la ciudad, que dejaba constancia del desencuentro que ello suponía. (Fig. 6)

En los años de 1960-70, el crecimiento del turismo de costa, consolidó el Paseo Marítimo como el instrumento urbano que definía el encuentro de la ciudad con el mar, estableciéndose como espacio intermedio de

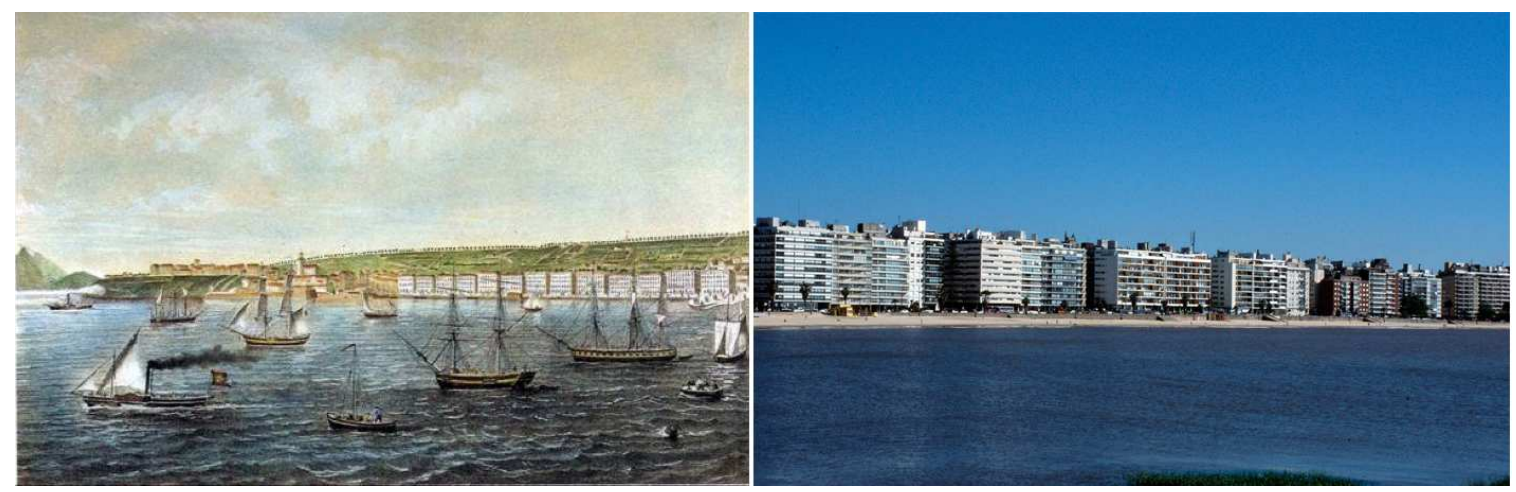

Fig. 5. Fachada Marítima: Casas del Muelle de Santander en el siglo XIX y Barrio de Pocitos de Montevideo en el siglo $\mathrm{XX}$ 


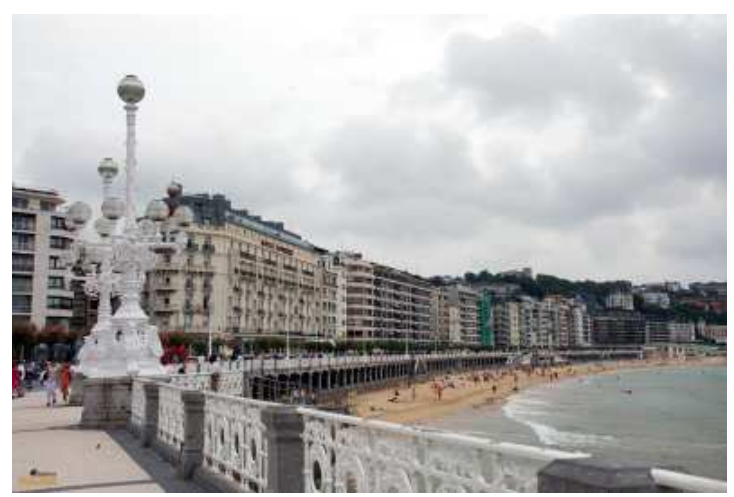

Fig. 6. Paseo marítimo de San Sebastián.

esa relación.

Ahora el paseo marítimo aunque sigue manteniendo su planteamiento inicial de recorrido lineal frente al mar, paralelo a la línea de costa, sin embargo plantea la secuencia de espacios, con cierta diversidad, creando remansos con arbolado, o zonas tensionadas donde el diseño del pavimento es clave en la definición de los flujos de ciudadanos.

Roberto Burle Max, como artista plástico y arquitecto paisajista, definió sus obras a partir de la idea de que el arte del paisaje es el arte de dirigir la mirada, modificando su percepción, estableciendo una armonía natural, recuperando especies autóctonas desconocidas, convirtiendo los espacios en paisajes espontáneos, y sobre todo muy humanos.

En Copacabana muestra la idea de que el mar, el paisaje no es solo un objeto de contemplación,

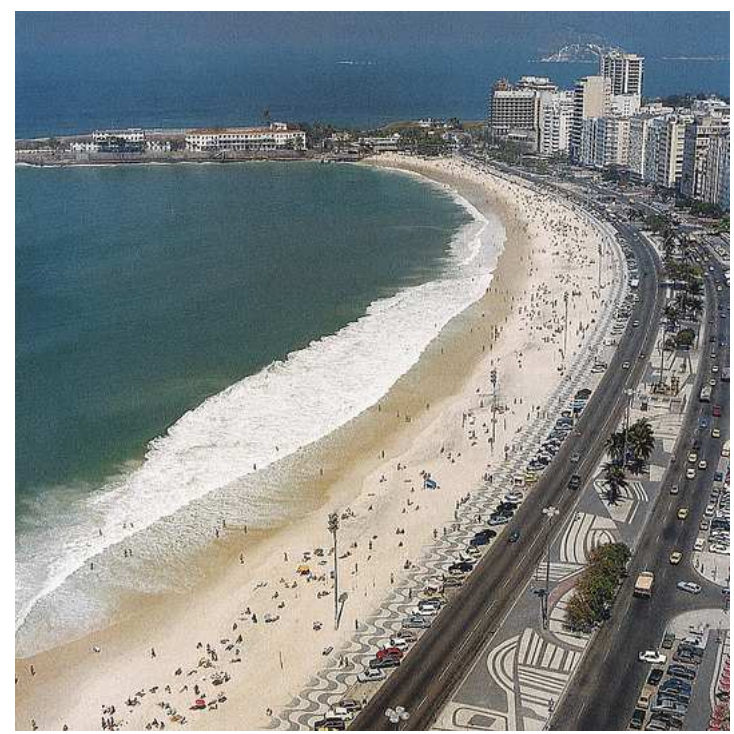

Fig. 7. Paseo marítimo de la Playa de Copacabana. Roberto Burle Max 1970 sino que tiene una función social. Aquí es el peatón el que domina la escena, a través de un pavimento duro, de mosaico portugués de piedra caliza, con composiciones abstractas que definen lugares, tensiones, recorridos, donde el árbol domesticado escultóricamente establece el remanso, la estancia. (Fig. 7)

\section{Diálogo transversal ciudad-mar:}

Pero en las ultimas décadas, se han planteado mecanismos de interacción ciudad-mar, buscando un diálogo transversal naturalezaartificio, intentando con ello conseguir que el tejido urbano se diluya en la playa-mar.

Uno de los recursos arquitectónicopaisajísticos utilizados para conseguir esa interacción, ha sido la continuidad del plano público, creando incluso espacios protegidos ante la fuerza del mar, como es el caso del paseo marítimo Juan Aparicio de Torrevieja, realizado por Carmen Pinós en 2000. (Fig. 8)

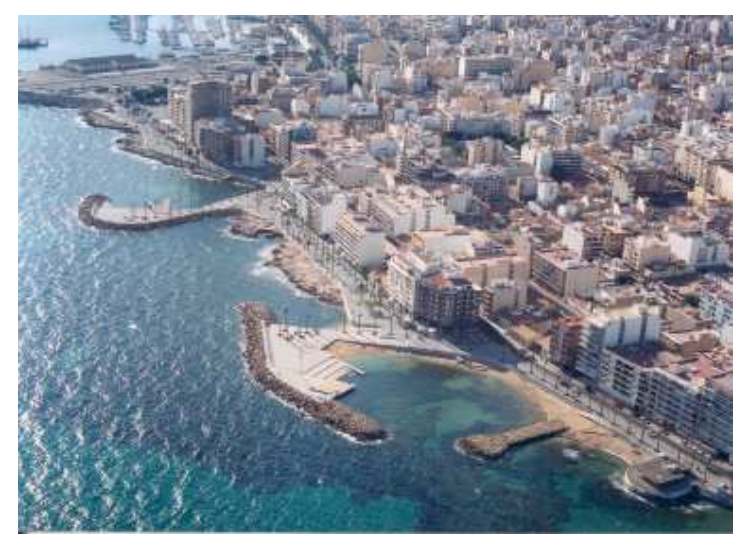

Fig. 8. Paseo marítimo Juan Aparicio de Torrevieja. Carmen Pinós, 2000.

Aquí el paseo marítimo, deja de ser lineal, paralelo a la línea de costa, para convertirse en una secuencia de espacios de oportunidad, que interaccionan tanto con la ciudad como con el mar.

Cuando la relación ciudad-mar, se produce en playas de arena en las que el plano de la ciudad se sitúa a la misma cota que la arena, esa interacción se consigue mediante la disolución del plano-suelo hasta confundirse con la arena, como lo realizó Olga Tarrasó en el frente marítimo de la Barceloneta en 1990, o bien en 


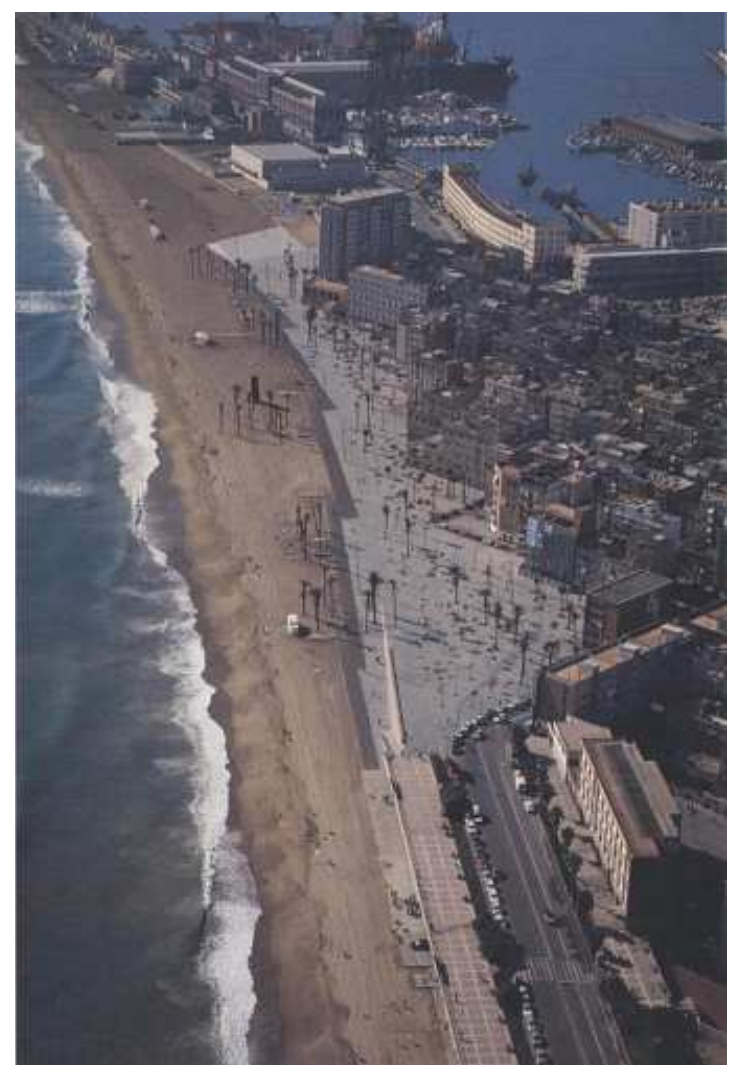

Fig. 9. Frente Marítimo de la Barceloneta. Olga Tarrasó y Espinás. 1990.

2012 en el paseo marítimo de Badalona (Fig. 9)

En estos dos paseos, es de nuevo clave el diseño y materiales del pavimento que conforma el plano, de forma que la disposición a $45^{\circ}$ de las losas pétreas, siguiendo la dirección del tejido urbano, tensionan el conjunto del espacio introduciéndole un fuerte dinamismo. La iteración con la arena, se consigue de forma suave a través de un pavimento intermedio, mas amable como es la madera.

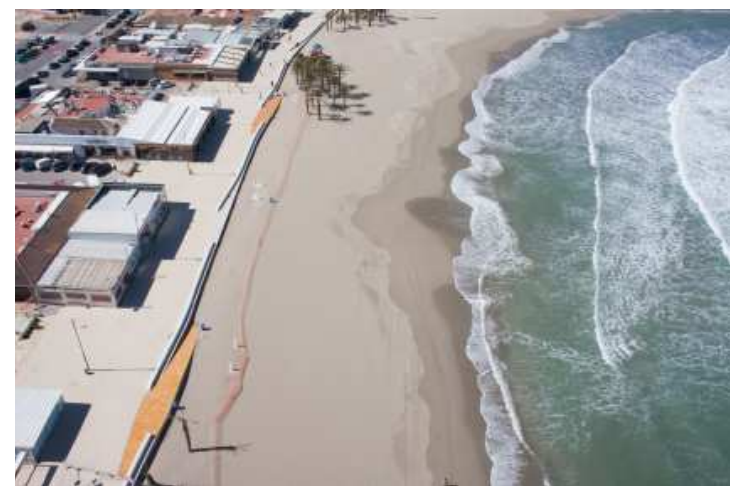

Fig. 10. Paseo del Arenal, Jávea. Alicante. M. Lillo y E. Vicedo, 2013
Este mismo recurso de la continuidad del plano de la ciudad, es el que utilizan Manuel Lillo y Emilio Vicedo en el Paseo del Arenal en Jávea (Alicante), en el 2013. Las diferencias se establecen en la búsqueda de un plano neutro, no direccional, con un pavimento repetitivo, que tensiona en todas las direcciones por igual, y especialmente en la forma escultórica del diseño de los bancos serpenteantes de hormigón y de las lenguas de conexión con la arena, también aquí de madera. (Fig. 10)

Pero no siempre la relación del plano suelo de la ciudad puede conectarse con el mar en la misma cota, ya que la fuerza de las crecidas de las mareas en determinados puntos, han convertido esa relación en un tema aparentemente insalvable.

Es el caso del frente de costa del pueblo de Hjerting en Dinamarca, en el que la playa había estado siempre separada de la ciudad mediante un muro y una barrera de protección de grandes piedras.

Una simple operación de disponer sobre esa barrera, un entablado de madera ha establecido

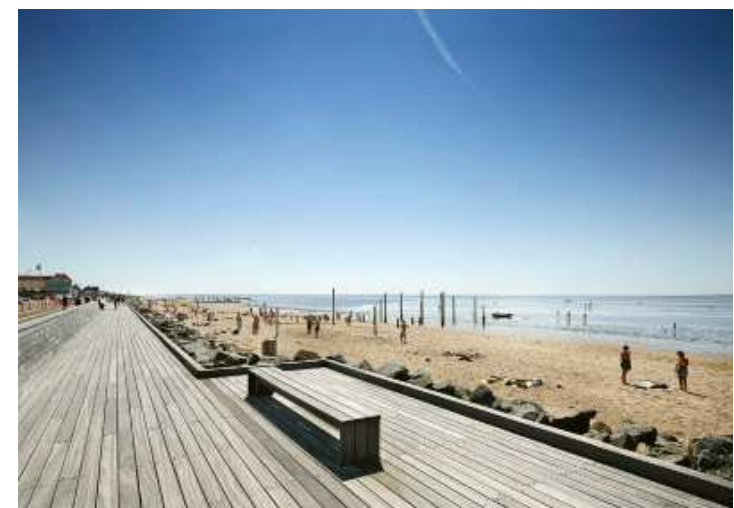

Fig. 11. Paseo de Esbjerg. Dinamarca. Nathan Romero \& Spektrum Arquitectos. 2011

una fuerte relación ciudad-mar, creando las condiciones para una intensa interacción social, a la vez que una posible acción contemplativa, mediante la chaislong de $700 \mathrm{~m}$ de longitud, que permite reencontrar el horizonte

En esta promenade, se exploran las posibles relaciones entre la tierra firme y el mar, de forma que la nueva plataforma se conecta con el agua creando diversos acontecimientos como la piscina marítima, el muelle flotante, o el propio paseo. (Fig. 11) 
En la buscada relación de continuidad del plano del espacio urbano público de la ciudad hacia el mar, cabe plantear también recursos de iteración directa con el agua, de forma que sea esta la que tome protagonismo en función de su cambiante disposición por las mareas.

Es el caso del frente marítimo del Puerto Jack Evans de Australia, donde la unión ciudad-mar se produce a través de una secuencia de zona parque verde - plano intermedio de madera $-\mathrm{y}$ línea fragmentada de borde de hormigón que se intersecta directamente con el mar, creando un espacio de ocio con gran diversidad de opciones en contacto con el agua como: playa, terraza, espigón de rocas, embarcadero urbano, paseo de madera, anfiteatro en el agua, zonas para bañarse y nadar, puntos de pesca, .....

La sencillez del diseño, realizado con sólidas piezas prefabricadas de hormigón, muestran su capacidad para soportar las inundaciones derivadas de las mareas, estableciendo un diálogo permanente con la fuerza del mar. (Fig. 12)

Por ultimo en este recorrido de la evolución del encuentro de la ciudad con el mar, cabe señalar actuaciones que buscan conseguir ese encuentro, recuperando los valores naturales y paisajísticas del litoral.

La propuesta en el frente marítimo de Castelldefels, trata de establece orden en un frente densamente ocupado por edificaciones que conforman una barrera entre la ciudad y el mar. Y ello lo hace a través de la disposición de una secuencia de ejes transversales que surgiendo de la prolongación de las calles se internan en la playa, estableciéndose como espacios de encuentro y cosido entre el medio urbano y el natural.

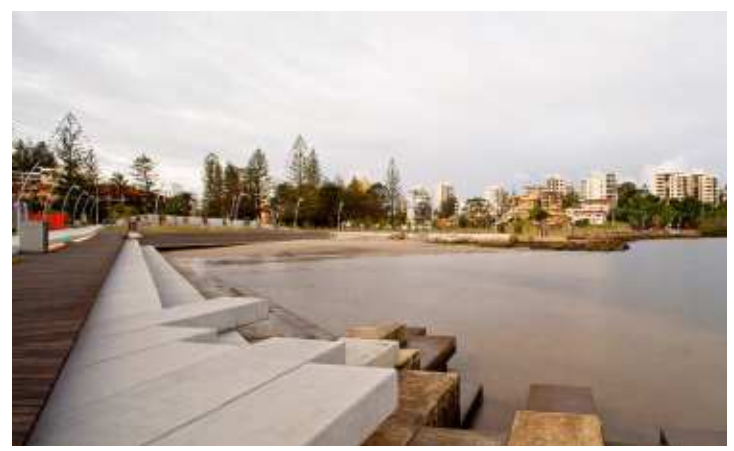

Fig. 12. Frente marítimo del Puerto Jack Evans en Australia. 2011.

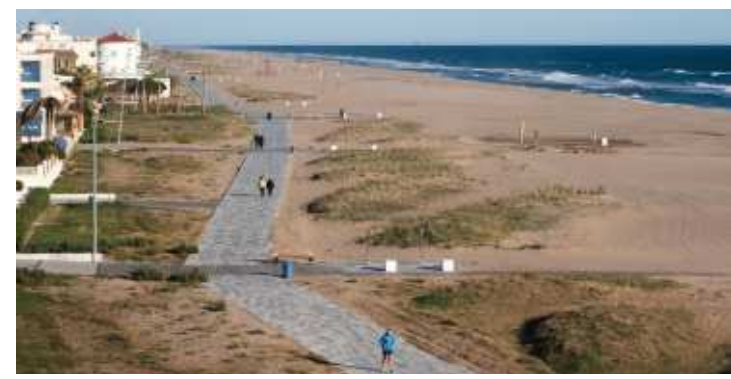

Fig. 13. Frente marítimo Castelldefels. Enrica Fontana-Xavier Nogués Arquitectos. 2009

Estas conexiones transversales, planteadas con vegetación autóctona y pavimento de madera, se conectan atravesando, un nuevo paseo, separado de las edificaciones, que a modo de vereda sinuosa, se posa sobre la arena como elemento autónomo, que no pertenece ni a la ciudad ni al mar, por lo que actúa de elemento de transición en esa relación.

El paseo está realizado por grandes losas prefabricadas de hormigón, que se apoyan sobre una base de confinamiento celular de polietileno de alta densidad, que proporciona una base rígida muy resistente a la flexión.

Esta actuación está permitiendo regenerar los ecosistemas perdidos, recuperando el sistema de dunas originario. (Fig. 13)

En definitiva este planteamiento de respecto por la naturaleza, no es algo nuevo, pues ya en 1932-34 Arne Jacobsen lo planteó en su actuación en la Playa de Bellavista, donde el diseño y el material empleado, la madera, supuso una clara integración artificionaturaleza.

\section{Conclusión}

Lo aquí expuesto muestra una evolución de la relación ciudad-mar claramente positiva al pasar de un planteamiento invasivo de la ciudad sobre el espacio natural modificando su ecosistema, a un diálogo transversal, de forma que la ciudad y la naturaleza, en este caso el mar, se complementen estableciendo una integración secuenciada, donde el tejido urbano se diluye como si de una mancha de aceite se tratara. Ello está suponiendo la regeneración de los ecosistemas perdidos a la vez que una nueva mirada de la sociedad hacia la naturaleza, teniendo mucha mas conciencia de su alto valor ecológico. 


\section{Referencias}

Aspect Studios. Puerto Jack Evans. Tweed Heads. Australia. Paisea: revista de paisajismo, ISSN 1887-2557, No. 26, 2013 (Ejemplar dedicado a: La costa), págs. 48-53

CARMONA, L., GÓMEZ, M. Montevideo: proceso planificador y crecimientos [en línea]. Montevideo: Udelar. FARQ. IHA. 2002

FONTANA, E.; NOGUÉS, X. AMB arquitectos. Tratamiento ambiental del frente marítimo. Castelldefels. España. Paisea: revista de paisajismo, ISSN 18872557, No. 26, 2013 (Ejemplar dedicado a: La costa), págs. 38-43

IWATA, N.; DEL RIO, V.: The Image of the Waterfront in Rio de Janeiro, Urbanism and Social Representation of Reality, En: Journal of Planning Education and Research 24: S. 171-183; Association of Collegiate Schools of Planning, 2004

REY PÉREZ, J. La Documentación de la intervención de Burle Marx en el Paseo de Copacabana: lecturas para la protección de un patrimonio paisajístico moderno. $9^{\circ}$ seminário docomomo brasil interdisciplinaridade e experiências em documentação e preservação do patrimônio recente brasília . junho de 2011 . www. docomomobsb.org

ROMERO, N.; Arkitekter \& Spektrum Arkitekter. Paseo marítimo de Esbjerg. Dinamarca. Paisea: revista de paisajismo, ISSN 1887-2557, No. 26, 2013 (Ejemplar dedicado a: La costa), págs. 20-25

PINOS, C. Paseo marítimo Juan Aparicio y acondicionamiento de la costa. España. Via arquitectura, ISSN 1137-7402, $\mathrm{N}^{\mathrm{o}}$ 6, 1999, pág. 70

TARRASÓ CLIMENT, O.; ESPINÁS CASAS, J. Paseo Marítimo de Badalona. Barcelona. On diseño, ISSN 1695-2308, N N $^{\circ} 335$ (El número correcto sería el 335/336), 2013

TERRY, T.: Praia de Copacabana: O espaço do Carioca - História, Forma, Usos e Significados, Master Thesis, PROURB, FAU, UFRJ, Rio de Janeiro, 2002

Notas manuscritas de Serapio Múgica sobre autoridades de la provincia de Gipuzkoa.

Plano de la ciudad de San Sebastian : 1900.
Plano de 18 × $27 \mathrm{~cm}$ en h. de 20 × $28 \mathrm{~cm}$; col. Archivo General de Gipuzkoa $=$ Gipuzkoako Artxibo Orokorra. Signatura: AGG-GAO : JD SM 47,6 